\title{
Memory Machines: Exploring how Digital and Wearable Technologies can be used to Visualise the Phenomena of Memory and the Concept of Identity
}

\author{
Dalia Yassine \\ Researcher \\ Learning Technology Research Centre (LTRC) \\ Ravensbourne University \\ London, UK
}

\section{OVERVIEW}

This research looks at how digital and wearable technology can be used to visualise the phenomena of memory in order to understand the evolving concept of identity.

Memory is one of the most important factors dictating the human condition and creative expression. Nearly everything we view, assess and relate to, is dependent on the phenomena of memory. We are surrounded by an amalgam of memories which spread over time and space, leaving traces of fragmented perceptions and dispersed information.

Artists, writers, philosophers and scientists have not ceased in the attempt to tackle, illustrate, visualise and interpret the widespread concept of memory and its relationship to identity through a diverse range of approaches and media.

When contemplating the nature of our identity, one turns to his or her thoughts, memories and sensations. By doing so, we reignite our internal connections and mental maps. Different 'memory branches' are activated to achieve some sort of final visualisation. These diverse branches and trajectories act as internal landscapes in which culture, nature, and history, have been imprinted.

All these elements are Memory Machines - we are what we remember. Henri Bergson (2004) in his Matter and Memory articulates, 'there is no perception which is not full of memories". Memory functions in every act of perception, in every act of intellection, in every act of language.
Memory by definition is the recalling of a certain situation as it was in itself but when in the act of recalling one can easily 'recreate' certain elements (altering details, feelings...), which leads to a misrepresentation of that memory. So in that case, is it actually the same or even a 'memory' at all? This repetitive and 'quasi-neurotic' condition appears to take on the role of a collective therapy leading art to become an object of transfer (i.e., a transfer of experience, perception or identity/ identification). The core question of this research is can ambient technology ensure memory is correctly encoded to enable a persistence of authentic identity?

Keeping in mind that the reality of the past resides in the artefacts of its representation (Hutton 1993) we are constantly in the act of reconstructing the past whilst anticipating the likely life trajectory of our future. Technological intervention into nature, notes Giddens (1991), is a condition of the development of abstract systems. Perhaps what we are ultimately doing with these so-called memory machines, is crafting alternate personas in virtuality, which could be seen a defining factor for our contemporary society. Is this a sort of escapism or, possibly, just a modern manifestation of something we have always pursued?

The phenomena of memory stands as selfreflexive, open ended, indeterminate, and forever incomplete. Having tried to represent memory via different artistic and mediated outlets, we are constantly in the loop of some sort of 'resymbolising' what is already symbolic. 
What effect does this have on our notions of identity? How can visualisation and wearable technology make this process more concrete and meaningful?

\section{REFERENCES}

Bergson, H. (2004) Matter and Memory. Courier Corporation.
Giddens, A. (1991) Modernity and Self-identity: Self and society in the late modern age. Stanford University Press.

Hutton, P. H. (1993) History as an Art of Memory. UPNE. 